\title{
Excitation-Contraction Coupling between Human Atrial Myocytes with Fibroblasts and Stretch Activated Channel Current: A Simulation Study
}

\author{
Heqing Zhan and Ling Xia \\ Department of Biomedical Engineering, Zhejiang University, Hangzhou 310027, China \\ Correspondence should be addressed to Ling Xia; xialing@zju.edu.cn
}

Received 2 May 2013; Revised 13 July 2013; Accepted 13 July 2013

Academic Editor: Zhonghua Sun

Copyright (C) 2013 H. Zhan and L. Xia. This is an open access article distributed under the Creative Commons Attribution License, which permits unrestricted use, distribution, and reproduction in any medium, provided the original work is properly cited.

Myocytes have been regarded as the main objectives in most cardiac modeling studies and attracted a lot of attention. Connective tissue cells, such as fibroblasts (Fbs), also play crucial role in cardiac function. This study proposed an integrated myocyte- $I_{\text {sac }}{ }^{-}$ $\mathrm{Fb}$ electromechanical model to investigate the effect of $\mathrm{Fbs}$ and stretch activated ion channel current $\left(I_{\mathrm{sac}}\right)$ on cardiac electrical excitation conduction and mechanical contraction. At the cellular level, an active Fb model was coupled with a human atrial myocyte electrophysiological model (including $I_{\text {sac }}$ ) and a mechanical model. At the tissue level, electrical excitation conduction was coupled with an elastic mechanical model, in which finite difference method (FDM) was used to solve the electrical excitation equations, while finite element method (FEM) was used for the mechanics equations. The simulation results showed that Fbs and $I_{\text {sac }}$ coupling caused diverse effects on action potential morphology during repolarization, depolarized the resting membrane potential of the human atrial myocyte, slowed down wave propagation, and decreased strains in fibrotic tissue. This preliminary simulation study indicates that $\mathrm{Fbs}$ and $I_{\mathrm{sac}}$ have important implications for modulating cardiac electromechanical behavior and should be considered in future cardiac modeling studies.

\section{Introduction}

Heart is considered as a composite material consisting of myocytes, Fbs, endothelial, vascular smooth muscle, neuronal cells, and fluids $[1,2]$. The division and interaction of these cells and fluids keep the heart working efficiently, which is performed by a well-ordered interplay between cardiac electrophysiology, excitation propagation, and force development. The process from the electrical excitation of the myocyte to mechanical contraction is referred to as cardiac excitation-contraction coupling (ECC). Conversely, changing the cardiac mechanical environment to alter electrical activity is referred to as mechanoelectric feedback (MEF) [3]. Investigating the multiphysics and multiscale heart system involves extremely difficult experiments in order to directly observe and manipulate the process that underlies cardiac electrical and mechanical activity. To overcome these experimental challenges, some sophisticated mathematical models have been developed to gain a better insight [4-9].
To study cardiac ECC, many electromechanical (EM) models, from the molecular level of myofilaments (MFs) to the anatomy of the organs, have been developed under both normal and pathological situations [6,10-14]. At the subcellular level, actin-myosin interaction and Ca-based activation are represented by MF models. Cellular reconstruction of electrophysiology and $\mathrm{Ca}$ handling are then coupled with MF models to produce EM cell models. At the organ level, electrical component is solved as a reaction-diffusion system and mechanical component is described by equations of continuum mechanics [4].

For cardiac MEF, the main focus of published studies has been placed on the function of stretch activated channels (SACs) in various noncardiac tissues $[15,16]$ as well as in the heart $[17,18]$. The electrophysiologic effects of pulsatile stretch, stretch generated by increased preload and afterload, and acute static mechanical stretch can be explained by SAC current $\left(I_{\mathrm{sac}}\right)$. 
The published models of ECC and MEF mainly described the properties of cardiac myocytes. However, the vasculature and connective tissue cells [19] have not been considered. Recently, it has been revealed that Fbs are numerously present in cardiac tissues with much smaller size than that of myocytes [20], and they affect the restitution properties of cardiac tissues, especially during the process of ageing and in various cardiac diseases [21-23]. Once the percentage of $\mathrm{Fbs}$ in the heart increases up to $10-35 \%$, a remodeling of cardiac structure occurs, which increases muscle stiffness and reduces the coupling between adjacent muscle fiber bundles [24]. The functional roles of Fbs on cardiac electrical and mechanical activities have attracted more and more interests. Both experimental and computational studies [2527] have confirmed that increased $\mathrm{Fb}$ population could lead to nonmonotonic changes in the conduction velocity and implicated that the effects of Fbs on cardiac electrophysiology and mechanics are worthy of further investigation.

Although the myocytes have been regarded as the main objectives for the cardiac electrophysiological, mechanical, and EM models [5-8], the role of Fbs has also been investigated recently using the models. By including the Fbs in the cardiac electrophysiological simulations [1,27-29], it has been reported that myocyte-Fb coupling modulated action potential (AP) morphology and action potential duration (APD). However, to our best knowledge, due to the lack of experimental data and accurate models of $\mathrm{Fb}$ mechanics, the myocytes and Fbs have not yet been integrated to investigate their electromechanics interaction. The aim of this study was to propose an integrated myocyte- $I_{\text {sac }}-\mathrm{Fb}$ electromechanical model to investigate the effect of Fbs and stretch activated ion channel current $\left(I_{\mathrm{sac}}\right)$ on cardiac electrical excitation conduction and mechanical contraction. The proposed model integrated the coupling between the Fb model [30] and the myocardial electrophysiological model [31] (including $I_{\mathrm{sac}}$ [32]) and mechanical model [33] at the cellular level, with excitation conduction and elastic mechanics [34] at the tissue level. The developed model was validated by comparing the simulated results of excitation conduction and mechanical properties to those from previous electromechanical coupling models. Electromechanical models of central point stimulus and strain maps were illustrated to investigate the effects of Fbs and $I_{\mathrm{sac}}$ on cardiac excitation conduction and mechanical contraction.

\section{Materials and Methods}

The framework of the coupled myocyte- $I_{\text {sac }}-\mathrm{Fb}$ model includes two parts. The first part includes the electrophysiological and mechanical models of myocytes and Fbs at the cellular level. The cell model stems from the CourtemancheRamirez-Nattel (CRN) model of the human atrial AP [30] (including $I_{\text {sac }}$ equations described by Kuijpers et al. [31]), the Rice mechanical model [32], and the Maleckar model of atrial Fbs [33]. The second part includes the models of excitation conduction and contraction of the Fb-myocyte coupling (FMC) at the tissue level. The tissue model includes cardiac excitation conduction and finite deformation, described by the Nash model [34]. Details of the individual components and their modification from published models are described as follows.

\subsection{Myocyte- $I_{\text {sac }}$-Fb Electrophysiological Modeling at the Cellular Level}

2.1.1. Electrophysiological Model of the Human Atrial Myocyte and $I_{\text {sac }}$. The CRN model was employed in this study, which can accurately represent atrial AP dynamics [30]. This computationally efficient model was based on the experimental data and reproduced various AP behaviors. Equations of $I_{\mathrm{sac}}$ were described by Kuijpers et al. in [31]. In their model, the amount of $I_{\text {sac }}$ was related to local stretch and can be changed during contraction.

2.1.2. Electrophysiological Model of Atrial Fbs. For the electrophysiological model of atrial Fbs, the active 1 model described by Maleckar et al. was applied in our study [33].

2.1.3. Cell-Cell Electrophysiological Coupling. The electrophysiological FMC is expressed as [33]

$$
\begin{aligned}
& \frac{d V_{\text {myo }}}{d t} \\
& =-\frac{\left(1 / C_{m, \text { myo }}\right)}{\left[I_{\text {myo }}\left(V_{\text {myo },} t\right)+I_{\text {stim }}+\sum_{i=1: n} G_{\text {gap }}\left(V_{\text {myo }}-V_{\mathrm{Fb}}\right)\right]} \\
& \frac{d V_{\mathrm{Fb}}}{d t}=-\frac{\left(1 / C_{m, \mathrm{Fb}}\right)}{\left[I_{\mathrm{Fb}}\left(V_{\mathrm{Fb},},\right)-I_{\mathrm{gap}}\right]},
\end{aligned}
$$

where $V_{\text {myo }}$ and $V_{\mathrm{Fb}}$ are the transmembrane potential of the human atrial myocyte and the $\mathrm{Fb}$, respectively, $C_{m \text {,myo }}$ and $C_{m, \mathrm{Fb}}$ are the membrane capacitance of the myocyte and $\mathrm{Fb}$, respectively, and $I_{\text {myo }}$ and $I_{\mathrm{Fb}}$ are the net membrane current of the myocyte and $\mathrm{Fb}$, respectively. $I_{\text {stim }}$ is the stimulus current applied to the myocyte membrane, and $I_{\text {gap }}$ is the current that flows through the gap junction between the myocyte and each Fb. $G_{\text {gap }}$ represents the gap-junctional conductance.

\subsection{Myocyte- $I_{\text {sac }}-F b$ Mechanical Modeling at the Cellular} Level: The $\mathrm{Ca}^{2+}$-Force Relation. To simulate the mechanics of myocyte- $I_{\mathrm{sac}}-\mathrm{Fb}$ model, the $\mathrm{Ca}^{2+}$-force relation in myocyte and $\mathrm{Fb}$ was considered. In the present study, the computationally efficient Rice mechanical model was applied for the myocyte [32], which included phenomenological representations of both regulatory unit (RU)-RU cooperative interactions and a three-state model of crossbridge attachment and distortion [4]. In our study, this MF model was coupled to the CRN model by using the Ca concentration from the CRN model as the input to the MF model.

To the best of our knowledge, there has been no welldefined mathematical model to describe the tension in $\mathrm{Fb}$. The main reasons are as follows. Firstly, there is lack of experimental data due to the small size of individual Fbs [35]. 
Secondly, $\mathrm{Ca}^{2+}$ current, as a control factor of active tension, has not been identified for membrane ionic currents in $\mathrm{Fb}$ $[1,33,36,37]$. The active force of $\mathrm{Fb}$ was therefore ignored at the cellular level in this study.

2.3. Tissue Modeling. A two-dimensional (2D) cardiac tissue was represented by a rectangular grid of $225 \times 225$ points. Each point was regarded as a myocyte/Fbs complex. Fbs were coupled with each myocyte in two ways (longitudinal connection and lateral connection). Fbs distributed along the long axis of myocytes were referred as longitudinal connection, and along the short axis of myocytes was lateral connection. The electrical component was governed by the parabolic partial differential monodomain equation. The governing equations for the mechanical component were the stress equilibrium equations based on the large deformation theory [6]. The mechanical model proposed by Nash and Panfilov [34] was used, which included the stress equilibrium, the finite element approximations, the constitutive law, and the uniform isotonic boundary loads.

As the Fb-myocyte (F-M) ratio increases, the cardiac fibrotic remodeling leads to a progressive increase in cardiac passive stiffness $[38,39]$. The common approach to modeling ischemic myocardium is to change material descriptions of passive myocardium. In this study, the material constants in passive material properties were reevaluated to show the higher elastic stiffness of fibrotic tissue. Compared with the normal tissue (the material constants with units of stress were $2 \mathrm{kPa}$ and $6 \mathrm{kPa}$ ) [34], the material constants in fibrotic tissue were set to $4 \mathrm{kPa}$ and $12 \mathrm{kPa}$, respectively. In this way, the modulus of fibrotic tissue was doubled, within the range of experiment results [40].

2.4. Numerical Methods. The system was solved numerically using custom software written in Fortran language. At the cellular level, all state variables were updated by the forward Eular method. The FDM was applied to solve the reactiondiffusion equation. Following each time integration step, all parameters of cells were updated. The active stress was then interpolated at the four-node rectangle isoparametric element Gaussian points. Stresses of these active Gaussian points were served as the inputs to the governing equations of the tissue mechanics model. The stress equilibrium equation was solved by a nonlinear least square iteration method with different material constants from different tissues.

In the $2 \mathrm{D}$ coupling model, a tissue size of $225 \times 225$ grid points was used to study conduction velocity (CV) of plane wave propagation for longitudinal and transverse conduction. CV was calculated as the tissue length divided by the time interval between the time of stimulation and the time of the earliest excitation at the other end of the tissue. In this set of simulations, electromechanical activities and the change of strain due to a periodic point stimulation at the centre of the tissue were investigated. Central stimulus sites were chosen to be the smallest possible square regions $(5 \times$ 5 computational nodes), which made it possible to produce a propagating wave. Ten periodic central stimuli with a time interval of $500 \mathrm{~ms}$ were applied at the centre of the $2 \mathrm{D}$ tissue to ensure a stable excitation and contraction, and timing started when the 11th loop occurred. $I_{\text {sac }}$ has been considered. For FDM, the spatial resolution in the longitudinal direction was $0.1 \mathrm{~mm}$, while in the transverse direction was $0.04 \mathrm{~mm}$. The temporal resolution was $0.005 \mathrm{~ms}$. No-flux boundary conditions were used. For FEM, a mesh with $14 \times 14$ elements was used, which contained $16 \times 16$ grid points in plane wave and central point stimulus simulations. The temporal resolution for the mechanical model was $2 \mathrm{~ms}$. Strain maps of central stimulus were also investigated.

\section{Results}

3.1. Effects of $I_{\text {sac }}$ and Fbs on Atrial Myocyte AP. Figure 1(a) illustrates the AP of the human atrial myocyte coupled with $I_{\mathrm{sac}}$ for different stretch ratios $\lambda=1.1,1.2$, and 1.3 in comparison with the uncoupled control $(\lambda=1.0)$. With increasing $\lambda$ of 1.0,1.1,1.2, and 1.3, prolonged repolarization was observed. Their corresponding AP durations at 50\% repolarization $\left(\mathrm{APD}_{50}\right)$ were $189 \mathrm{~ms}, 196 \mathrm{~ms}, 197 \mathrm{~ms}$, and $202 \mathrm{~ms}$. When compared with control $(\lambda=1.0)$, an increase of $3.7 \%, 4.2 \%$, and $6.9 \%$ was obtained. At $90 \%$ repolarization, more obvious increase was found. AP durations $\left(\mathrm{APD}_{90}\right)$ were $372 \mathrm{~ms}$ and $435 \mathrm{~ms}$ for $\lambda=1.1,1.2$, with the increase of $21 \%$ and $42 \%$ in comparison with $307 \mathrm{~ms}$ from $\lambda=1.0$. With $\lambda=1.3$, the membrane potential was $-64.2 \mathrm{mV}$ at $500 \mathrm{~ms}$, and it did not return to $-69.5 \mathrm{mV}$ required for $\mathrm{APD}_{90}$. For the uncoupled control, the resting potential remained at $-81 \mathrm{mV}$ before the fast depolarization. For $\lambda=1.1,1.2$, and 1.3 , it depolarized slowly within $50 \mathrm{~ms}$ and increased by $4.9 \%$, $6.2 \%$, and $8.6 \%$ at $50 \mathrm{~ms}$ when compared with the uncoupled control. Therefore, it can be concluded that the coupling $I_{\text {sac }}$ prolonged repolarization and APD and depolarized the resting potential.

Figure 1(b) presents the repolarization for the human atrial myocyte when coupled with different numbers of Fbs $(1 \mathrm{Fb}, 2 \mathrm{Fbs}$, and $3 \mathrm{Fbs}$ ) in comparison with the control (no $\mathrm{Fb}$ ). With more coupled Fbs, the membrane potential during the plateau was less depolarized, leading to a $13 \%, 41 \%$, and $59 \%$ decrease (for 1,2 , and $3 \mathrm{Fbs}$ ) of $\mathrm{APD}_{50}$ when compared with control. However, for $\mathrm{APD}_{90}$, they were increased by $11 \%, 19 \%$, and $45 \%$. For the resting potential within $50 \mathrm{~ms}$, it increased by $2.5-6.2 \%$ as the number of coupled Fbs increased. Therefore, coupling Fbs decreased $\mathrm{APD}_{50}$, prolonged $\mathrm{APD}_{90}$, and depolarized the resting potential.

Similar changes were observed in myocyte AP with changed $G_{\text {gap }}$ but with fixed number of coupled Fbs. As shown in Figure 1(c), increasing $G_{\text {gap }}$ from 1 ns to 3 ns resulted in a $43-55 \%$ reduction of $\mathrm{APD}_{50}$, an $18 \%$ increase of $\mathrm{APD}_{90}$, and a $5 \%$ increase in the resting potential at $50 \mathrm{~ms}$.

Figure 1(d) shows the APs of four different coupling schemes. An uncoupled myocyte was referred to as control. The other three schemes were myocyte coupled with $I_{\mathrm{sac}}$ with $\lambda=1.1$, myocyte coupled with two Fbs with $G_{\text {gap }}=1 \mathrm{nS}$, and myocyte coupled with both $I_{\text {sac }}$ and two Fbs with $\lambda=1.1$ and $G_{\text {gap }}=1$ ns. It can be seen that $I_{\text {sac }}$ made the membrane potential during the plateau slightly more depolarized, while Fbs made it less depolarized. For $\mathrm{APD}_{50}$, the first scheme 


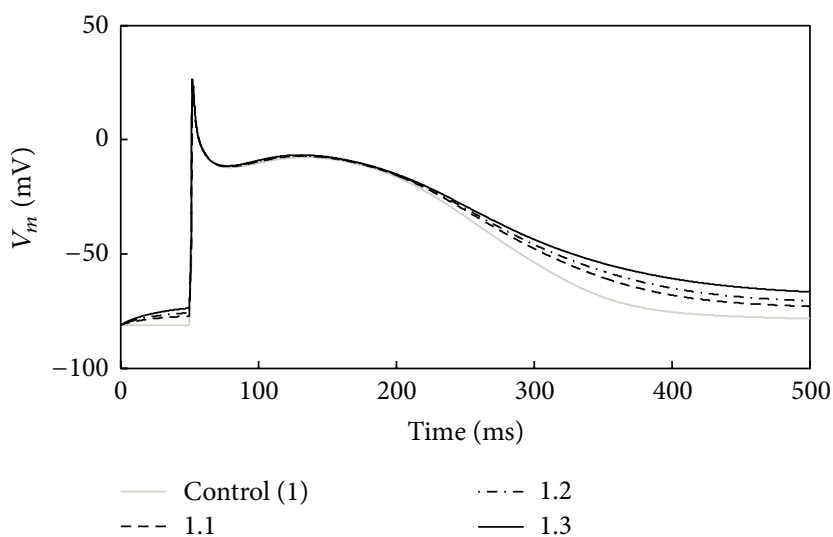

(a)

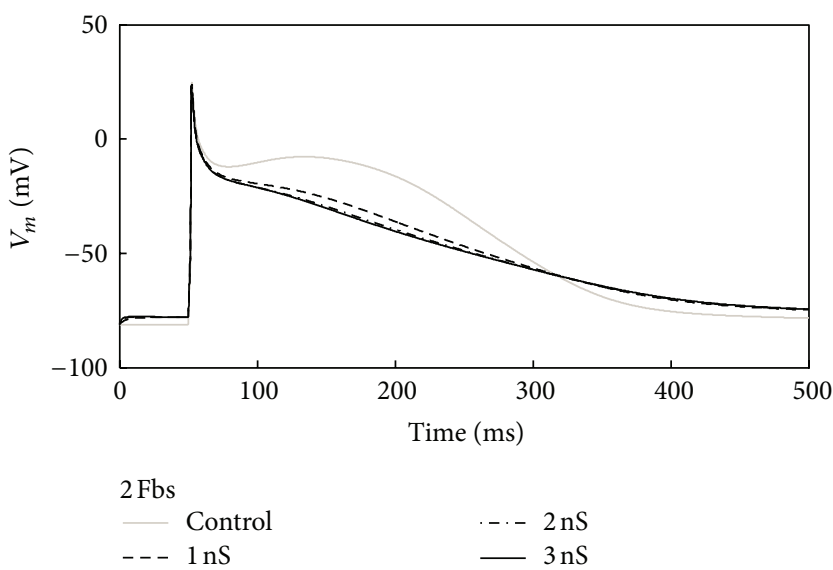

(c)

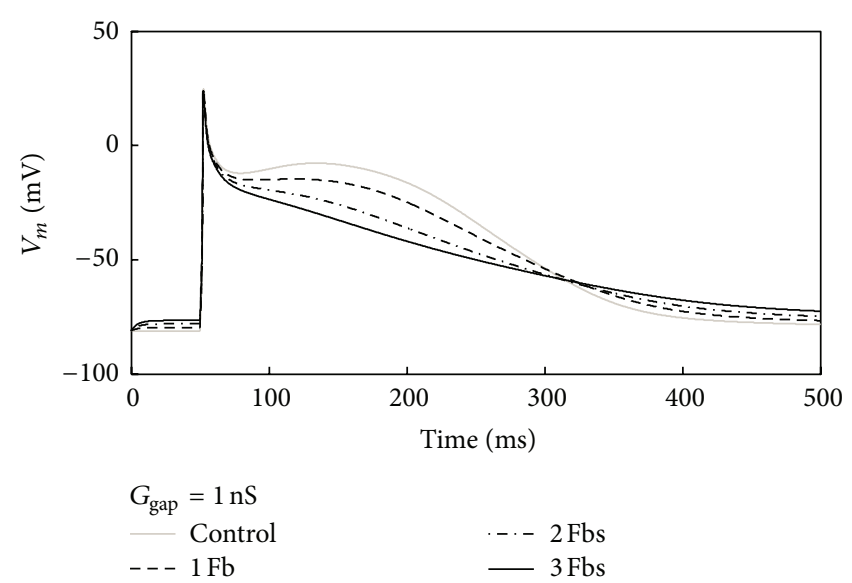

(b)

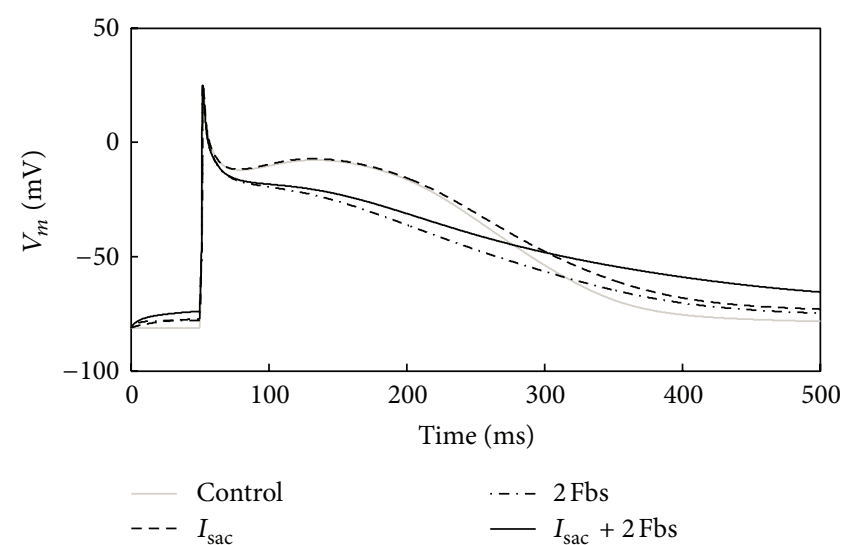

(d)

FIGURE 1: Illustration of changes in the waveform of the atrial myocyte AP. (a) 1 myocyte coupled to $I_{\text {sac }}$ with a $\lambda$ of 1.1 to 1.3 . (b) 1 myocyte coupled to 1 to $3 \mathrm{Fbs}$ with a $G_{\text {gap }}$ of $1 \mathrm{nS}$. (c) 1 myocyte coupled to 2 Fbs with a $G_{\text {gap }}$ of 1 to $3 \mathrm{nS}$. (d) 1 myocyte coupled to $I_{\text {sac }}$ or 2 Fbs or $I_{\text {sac }}$ and $\mathrm{Fbs}$ at the same time.

resulted in a 5\% increase and the second scheme resulted in a $41 \%$ decrease when compared with the uncoupled control. When the myocyte was coupled with $I_{\mathrm{sac}}$ and Fbs together, it was reduced by $38 \%$. For $\mathrm{APD}_{90}$, coupling only with $I_{\mathrm{sac}}$ or Fbs produced a $21 \%$ and $19 \%$ increase. However, for the case of $I_{\text {sac }}$ and Fbs together, the membrane potential was $-62.5 \mathrm{mV}$ at $500 \mathrm{~ms}$, higher than the required threshold for $\mathrm{APD}_{90}$. In addition, the resting potentials within $50 \mathrm{~ms}$ were all depolarized in the four coupling schemes. The case of coupling with both $I_{\mathrm{sac}}$ and Fbs had the highest increase of $9.8 \%$ when compared with the control. The increases in other two couplings were $\sim 5.5 \%$.

3.2. Effects of $I_{s a c}$ and Fbs on Atrial Myocyte $T_{a}$. As shown in Figure 2, with the same coupling schemes as described in Figure $1(\mathrm{~d})$ and with the cells stimulated at $1 \mathrm{~Hz}$, when compared to control, the peak of $T_{a}$ was slightly decreased by the coupled $I_{\mathrm{sac}}$ and decreased by $11 \%$ with the coupled Fbs. Coupling $I_{\mathrm{sac}}$ and Fbs together did not result in a further decrease in the peak in comparison with that from coupling

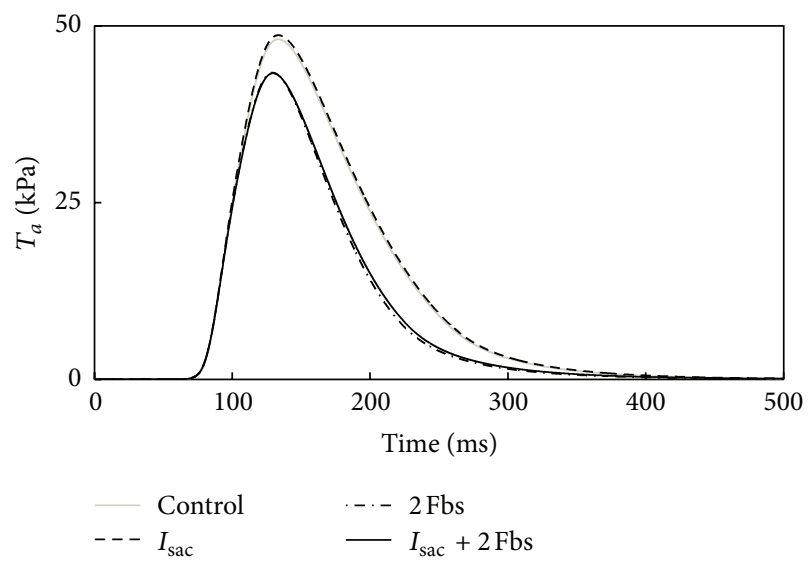

FIGURE 2: Illustration of changes in $T_{a}$ of the atrial myocyte after coupling to $I_{\mathrm{sac}}, 2 \mathrm{Fbs}$, or $I_{\mathrm{sac}}$ and Fbs at the same time.

Fbs only. In addition, coupling Fbs (whether coupling $I_{\mathrm{sac}}$ or not) accelerated the reduction of $T_{a}$. 


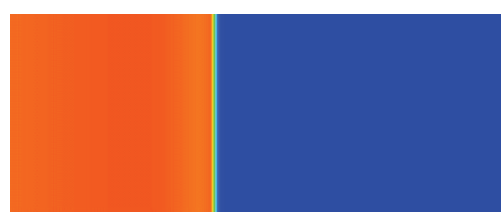

(a)

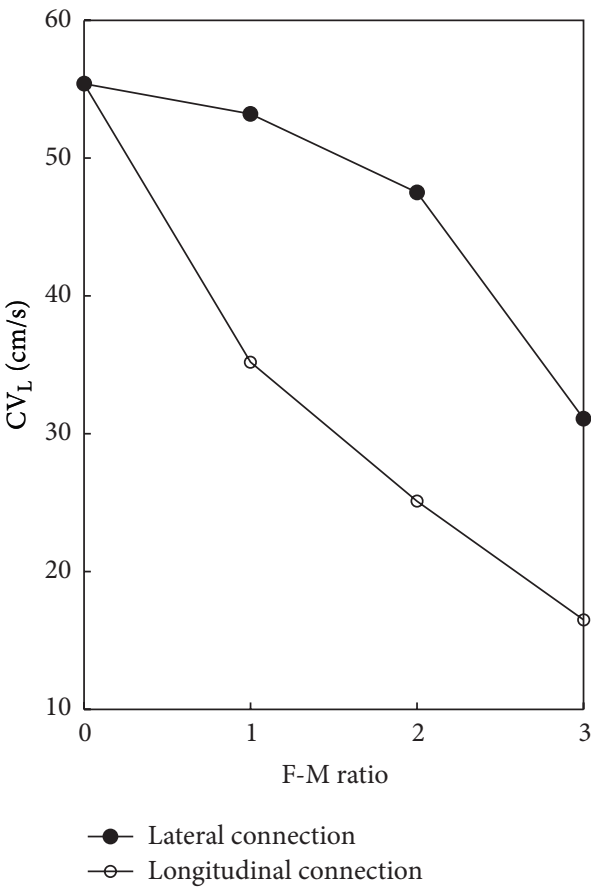

(c)

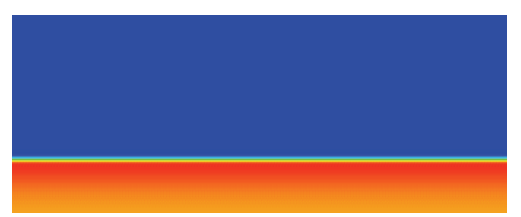

(b)

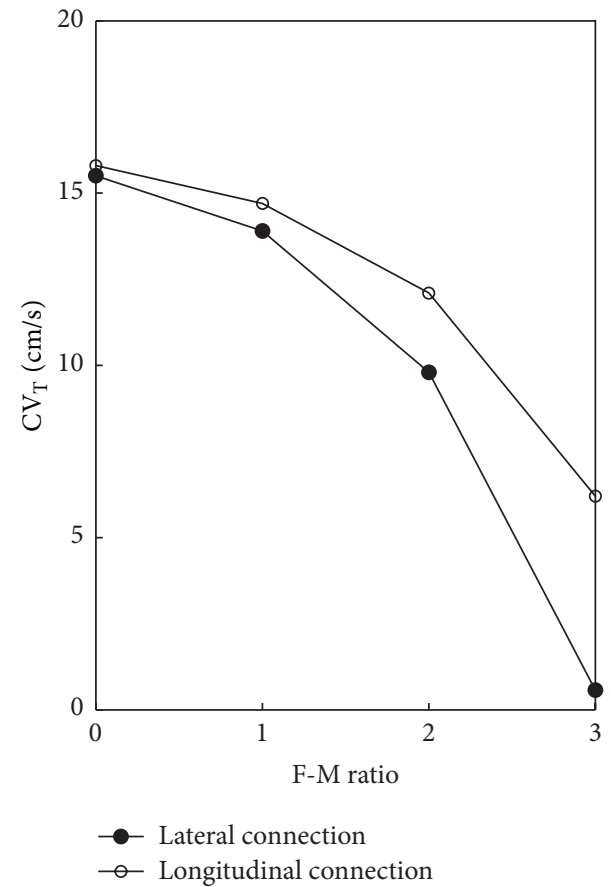

(d)

FIGURE 3: Effects of myocyte-Fbs coupling on CV: (a) longitudinal plane wave propagation, (b) transverse plane wave propagation. (c) $\mathrm{CV}_{\mathrm{L}}$ versus F-M ratio for longitudinal and lateral connections, and (d) $\mathrm{CV}_{\mathrm{T}}$ versus $\mathrm{F}-\mathrm{M}$ ratio for longitudinal and lateral connections.

3.3. Coupling Fbs Modulate Conduction Velocity. As shown in Figure 3, with the stimulation applied at the left end (for longitudinal conduction) or the bottom (for transverse conduction) of the tissue, it was observed that CV progressively decreased as the F-M ratio increased in either case of FMC. For longitudinal connection, Fbs were coupled at both ends of the myocyte, namely, Fbs distributed along the long axis. In this situation, with the F-M ratio from 1 to 3, longitudinal $\mathrm{CV}\left(\mathrm{CV}_{\mathrm{L}}\right)$ decreased by $37 \%$ to $70 \%$, and transverse $\mathrm{CV}\left(\mathrm{CV}_{\mathrm{T}}\right)$ decreased by $7 \%$ to $61 \%$. For lateral connection, Fbs were coupled along the lateral sides of a myocyte, with corresponding $\mathrm{CV}_{\mathrm{L}}$ decreased by $4 \%$ to $44 \%$, and $\mathrm{CV}_{\mathrm{T}}$ decreased by $10 \%$ to $96 \%$. From Figures 3(c) and $3(d)$, it can be seen that longitudinal connection resulted in a larger reduction in $\mathrm{CV}_{\mathrm{L}}$ than in $\mathrm{CV}_{\mathrm{T}}$, while lateral connection had a greater impact on $\mathrm{CV}_{\mathrm{T}}$.

3.4. 2D Simulation with Central Stimulus. Each grid in Figure 4(a) represents one atrial myocyte. Figure 4(b) gives one myocyte coupled with $2 \mathrm{Fbs}$ (with an F-M ratio of 2). Figure 4(c) represents one myocyte coupled with $3 \mathrm{Fbs}$ (with an F-M ratio of 3). $G_{\text {gap }}$ in Figures 4(b) and 4(c) was $1 \mathrm{nS}$. The type of FMC was lateral connection.
In electrophysiology, it can be seen that the depolarization wave was generated in the center and spread out over time. The shape of the start of depolarization was ellipsoid. As the number of coupling Fbs increased, the conduction of excitation wave slowed down. At $40 \mathrm{~ms}, \mathrm{CV}_{\mathrm{L}}$ from $2 \mathrm{Fbs}$ (Figure 4(b)) declined slightly when compared with the control (Figure 4(a)), but it declined significantly with 3 Fbs (Figure $4(\mathrm{c})$ ). $\mathrm{CV}_{\mathrm{T}}$ declined gradually as the $\mathrm{F}-\mathrm{M}$ ratio increased, which was consistent with the results in Figure 3. Coupling with Fbs produced flat depolarization wave. As the deceleration of excitation wave, the myocytes coupled with Fbs required more time to return to the resting state. At $370 \mathrm{~ms}$, tissues without $\mathrm{Fbs}$ and with an F-M ratio of 2 were at the resting state. However, the tissue with an F-M ratio of 3 did not reach the resting level even at $490 \mathrm{~ms}$. In addition, with the contribution from $I_{\mathrm{sac}}$ and Fbs, the resting potential in tissues coupled with Fbs was less negative than that in the uncoupled tissue.

In mechanics, with the $I_{\mathrm{sac}}$ and Fbs added in the tissue, the excitation in tissues was delayed, leading to the delay of $T_{a}$ in the same grid point in comparison with the uncoupled control. As a result, the deformation in coupled tissues lagged behind. At $150 \mathrm{~ms}$, the contraction was obvious in Figure 4(a), 
(a)

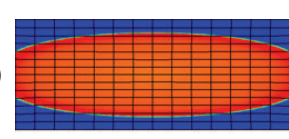

(b)

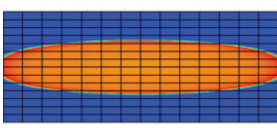

(c)

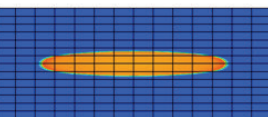

$40 \mathrm{~ms}$

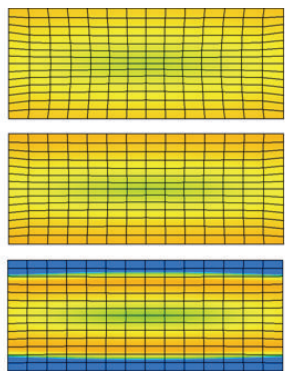

$150 \mathrm{~ms}$

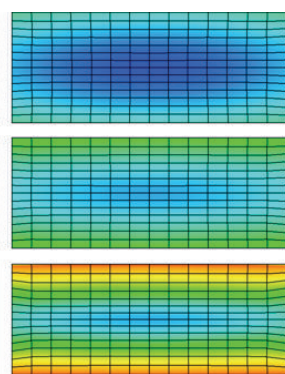

$260 \mathrm{~ms}$

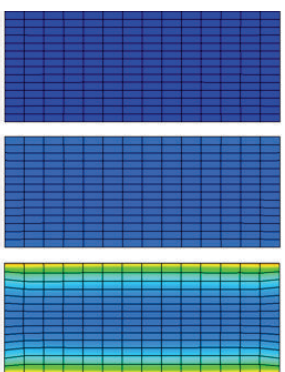

$370 \mathrm{~ms}$

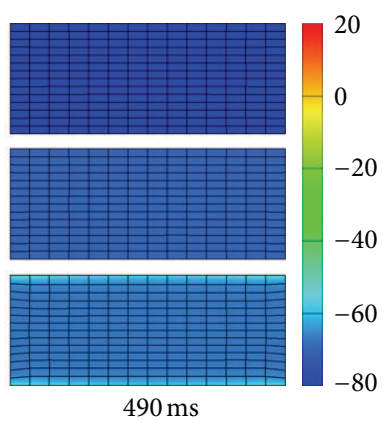

$490 \mathrm{~ms}$

FIGURE 4: Central stimulus simulation of electromechanical coupling: (a) myocyte cells only, (b) 1 myocyte coupled to 2 Fbs with lateral connection in each grid point, and (c) 1 myocyte coupled to $3 \mathrm{Fbs}$ with lateral connection in each grid point.

while imperceptible with $3 \mathrm{Fbs}$ (Figure $4(\mathrm{c})$ ). At $370 \mathrm{~ms}$ and 490 ms, meshes in Figures 4(a) and 4(b) gradually returned to relaxation along with the disappearance of propagating waves but kept contraction in Figure 4(c).

Figure 5 shows the quantitative deformations corresponding to the electromechanical activities in Figure 4. It shows that higher strains appear in the center area. In the uncoupled tissue, the maximum strain was 0.28 at $150 \mathrm{~ms}$. When the F-M ratio was 2 and 3, it declined to 0.22 and 0.17 , decreased by $21 \%$ and $39 \%$. As the F-M ratio increased, it took longer to reach the maximum. As shown in Figure 5(c), due to the delay of $T_{a}$, the strain reached the peak value at $260 \mathrm{~ms}$, 110 ms later than Figures 5(a) and 5(b). In addition, due to the slow conduction and less negative resting membrane in tissues with $\mathrm{Fbs}$, there were still slight deformations presented in these tissues at $490 \mathrm{~ms}$. Therefore, it can be concluded that coupling $I_{\mathrm{sac}}$ and Fbs delayed the deformation and decreased the maximum strain.

\section{Discussion}

The effects of coupling Fbs and $I_{\mathrm{sac}}$ on modulating human atrial myocyte excitability and AP morphology at the cellular level, and their effect on cardiac excitation wave conduction and contraction at the tissue level, have been investigated. The classical CRN model [30], the Rice model of the cardiac MF [32], the Maleckar model of atrial Fbs [33], and the Kuijpers model of $I_{\text {sac }}$ [31] were employed in this study. All these models were integrated as a myocyte- $I_{\mathrm{sac}}-\mathrm{Fb}$ coupling electromechanical model to investigate AP waveform and $T_{a}$ of human atrial myocyte as a function of $\lambda, G_{\text {gap }}$, and the number of Fbs. For tissue models, the influence of Fbs on plane wave propagation has been studied with the electromechanical activities and strain maps provided.

4.1. Role of $I_{\text {sac }}$ and Fbs Coupling on Human Atrial Myocyte Resting Membrane Potential, AP Waveform, and $T_{a}$. For $I_{\mathrm{sac}}$, Zabel et al. explored the electrophysiological effect of sustained stretch and reported that a sustained, static load of the isolated rabbit ventricle influences repolarization and activation. The disparity of stretch effects between various locations in the ventricle resulted in an increased dispersion of repolarization [41]. Franz et al. confirmed that atrial SAC was involved in the pathophysiology of atrial fibrillation $[42$, 43]. In this study, membrane potential of the myocyte coupled to $I_{\mathrm{sac}}$ with different $\lambda$ was investigated with a prolongation of repolarization and APD and a depolarization of the resting potential for $I_{\mathrm{sac}}$ coupling. Our results agreed well with the observation from Shaw and Rudy that increasing $\mathrm{K}^{+}$ concentration led to a depolarized membrane potential [44].

For Fbs, experimental research showed depolarization of neonatal rat ventricular cardiomyocyte strands when myofibroblasts interacted with myocytes [25]. Previous studies of FMC using myocyte-Fb coculture models have shown that the Fbs depolarized electrotonically coupled myocytes $[1,25,33,45]$. MacCannell et al. showed that the resting membrane potential of the coupled myocyte was depolarized slightly $(\sim 2.7 \mathrm{mV})$ for up to $10 \mathrm{Fbs}$ per myocyte and was insensitive to changes in $G_{\text {gap }}$ [1]. In the study by Maleckar et al. a $G_{\text {gap }}$ of $8 \mathrm{nS}$ and two active $1 \mathrm{Fbs}$ resulted in a resting membrane potential elevation of $8.3 \mathrm{mV}$ [33]. Our simulations also showed a depolarizing effect of coupled Fbs on resting membrane potential of the atrial myocytes. A maximum depolarization of $5.1 \mathrm{mV}$ was obtained for an Fb density of 2 Fbs per myocyte with a $G_{\text {gap }}$ of $1 \mathrm{nS}$ and a maximum depolarization of $3.9 \mathrm{mV}$ for a high $G_{\text {gap }}(3 \mathrm{nS})$ with an F-M ratio of 2.

Coupling the human atrial myocyte with Fbs in this study also resulted in diverse effect on AP morphology during repolarization. Maleckar et al. used the FMC model with a high $G_{\text {gap }}$ and found that $\mathrm{Fbs}$ functioned as strong current sources at rest and as both sources and sinks during the AP [33]. In their study, the prolongation of repolarization was early in the AP, and plateau was prolonged or shortened depending on both the $\mathrm{Fb}$ resting membrane potential and number of coupled Fbs [33]. MacCannell et al. compared ventricular AP when Fbs of $6 \mathrm{pF}$ or $60 \mathrm{pF}$ were coupled to a myocyte. The data showed that APD was shortened much more when large Fbs were coupled [1]. In our simulations, a myocyte coupled with different number of Fbs or different $G_{\text {gap }}$ resulted in less depolarization in the membrane potential during the plateau when compared with control. Meanwhile, increasing Fbs and $G_{\text {gap }}$ decreased $\mathrm{APD}_{50}$ and prolonged $\mathrm{APD}_{90}$.

Myocyte- $I_{\mathrm{sac}}-\mathrm{Fb}$ coupling has been considered to investigate the resting membrane potential and AP waveform 
(a)

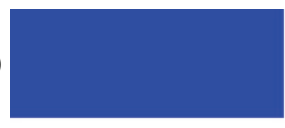

(b)

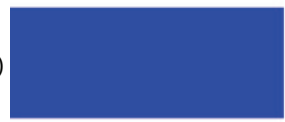

(c)

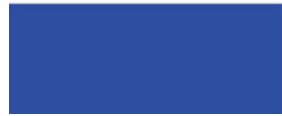

$40 \mathrm{~ms}$
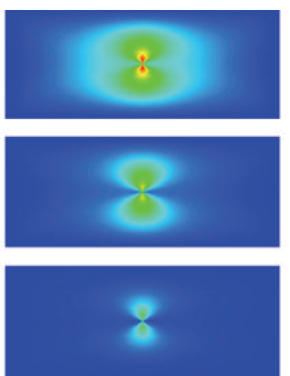

$150 \mathrm{~ms}$
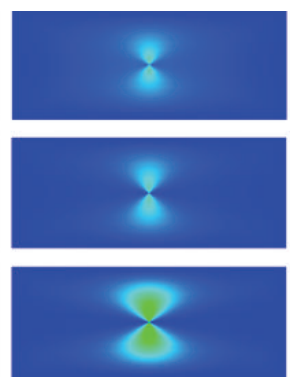

$260 \mathrm{~ms}$
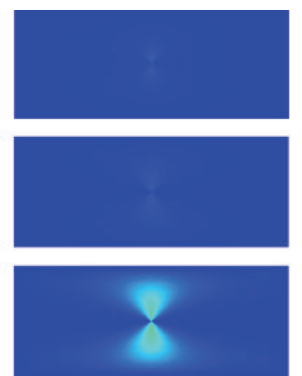

$370 \mathrm{~ms}$

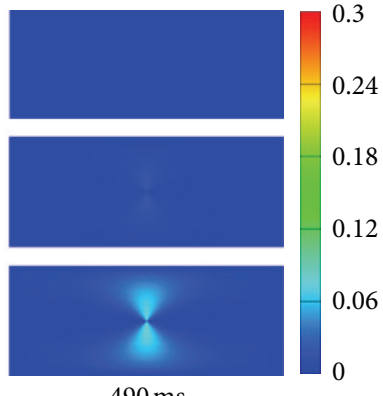

$490 \mathrm{~ms}$

FIGURE 5: Strain maps of the central stimulus simulation: (a) myocyte cells only, (b) 1 myocyte coupled to 2 Fbs with lateral connection in each grid point, and (c) 1 myocyte coupled to $3 \mathrm{Fbs}$ with lateral connection in each grid point.

of human atrial myocyte as a function of $\lambda, G_{\text {gap }}$, and the number of Fbs. Compared with only Fbs coupling or $I_{\text {sac }}$ coupling, the myocyte- $I_{\text {sac }}-\mathrm{Fb}$ coupling had the highest depolarization at the resting membrane potential. However, the reduction of $\mathrm{APD}_{50}$ in myocyte- $I_{\text {sac }}-\mathrm{Fb}$ coupling was between other two couplings.

In addition to the effect on the human atrial myocyte electrophysiology, coupled Fbs and $I_{\text {sac }}$ had important implications for the changes in $T_{a}$. In our study, $T_{a}$ had the maximum value of $48.6 \mathrm{kPa}$, which was consistent with the normal range (10-75 kPa) [46]. Kerckhoffs et al. computed myofiber contraction in both nonfailing and failing hearts. Their results showed that in the failing heart model, inotropy was decreased by reducing peak fiber $T_{a}$ by $27 \%$ [14]. In our study, the peak of $T_{a}$ was decreased $11 \%$ with the coupled Fbs. The reason was that $\mathrm{Fbs}$ coupling reduced the $\mathrm{Ca}$ concentration and caused a reduction in $T_{a}$. Due to the strong correlation between fibrosis and heart failure [47-49], it can be expected that once the number of Fbs increased, a significant reduction would be revealed in $T_{a}$. In contrast, $I_{\text {sac }}$ coupling did not bring much change in $T_{a}$.

4.2. Role of Fbs Coupling on CV. In an experimental study, Miragoli et al. found a biphasic effect on CV when endogeneous Fbs proliferated with myocytes [25]. In a modeling study, Xie et al. showed that Fbs, whether coupled to myocytes or not, slowed conduction by creating zigzag conduction pathways [23]. In our simulation, $\mathrm{CV}_{\mathrm{L}}$ and $\mathrm{CV}_{\mathrm{T}}$ were investigated in both longitudinal and lateral connections. The results revealed an unidirectional decreasing in $\mathrm{CV}$ with Fbs coupling, which was consistent with Xie et al. [23]. However, different coupling types had different influence on $\mathrm{CV}_{\mathrm{L}}$ and $\mathrm{CV}_{\mathrm{T}}$. For longitudinal connection, the conductance along the longitudinal direction dropped down, resulting in a significant decrease in $\mathrm{CV}_{\mathrm{L}}$. Similarly, lateral connection decreased the conductance along the transverse direction, which led to a higher reduction in $\mathrm{CV}_{\mathrm{T}}$.

4.3. Effects of $I_{\text {sac }}$ and Fbs Coupling at the Tissue Level. For central stimulus simulations, the effect of Fbs on cardiac excitation conduction and contraction has been investigated in this study. Nash and Panfilov presented the same electromechanical activity due to a periodic point stimulation at the center of the excitable medium [34]. Their modeling did not consider the biophysics of specific ionic currents and SAC channels. Meanwhile, $T_{a}$ has been directly modeled using a single ODE dependent on the transmembrane potential. In our study, specific cell electrophysiological and mechanical models of myocyte and $\mathrm{Fb}$ were included in the electromechanical modeling. Results in Figure 4 revealed that Fbs and $I_{\mathrm{sac}}$ coupling slowed down the conduction of excitation wave and depolarized the resting membrane potential, which confirmed results at the cellular level (Figure 1). Furthermore, due to the higher elastic stiffness of myocardial scar tissue [38-40], deformations in tissues coupled Fbs decreased by 21$39 \%$ when compared with uncoupled control (Figure 5). Our strain maps were similar to previous modelings and clinical data $[10,50]$. In [50], strains in normal regions were $-15.3 \pm$ $4.5 \%$ and $-10.6 \pm 5.3 \%$ in the infarct zone. Our calculation of strain was also in this range. On the basis of these results, we expected that once severe fibrosis occurred, strains decreased apparently due to the large stiffness in these areas, or similar to the rigid motion.

4.4. Limitations. Three limitations of this study should be mentioned. Firstly, the mechanisms of Fb mechanics should be further studied. It can be expected that once the detailed mechanical model of $\mathrm{Fb}$ is developed, it can be coupled to the myocyte mechanics model to simulate more precise mechanisms of myocyte- $I_{\text {sac }}-\mathrm{Fb}$ electromechanical coupling. Secondly, mechanosensitive currents have not been incorporated into the $\mathrm{Fb}$ model. However, previous studies implied that small electrical or mechanical perturbations in the cardiac $\mathrm{Fb}$ can alter the AP profile of the myocyte to which Fbs were coupled [51-53]. Thirdly, due to the lack of physiological data from the same species for the coupled model, only the existing experimental data relevant to each individual model was used to validate our simulation results.

\section{Conclusions}

In conclusion, a coupled myocyte- $I_{\text {sac }}-$ Fb electromechanical model has been developed by integrating an active $\mathrm{Fb}$ model with a human atrial myocyte electrophysiological model (including $I_{\mathrm{sac}}$ ) and a mechanical model. The effects of Fbs 
and $I_{\mathrm{sac}}$ coupling on cardiac excitation conduction and contraction have also been investigated. The simulation results confirmed that $\mathrm{Fbs}$ and $I_{\mathrm{sac}}$ coupling resulted in diverse effects on AP morphology during repolarization, depolarized the resting membrane potential of human atrial myocyte, slowed down wave propagation and decreased strains in fibrotic tissue, and indicated that Fbs and SACs are important mechanisms in electromechanical coupling and should be considered in cardiac electromechanical modeling.

\section{Acknowledgments}

This project is supported by the 973 National Basic Research \& Development Program (2007CB512100) and the National Natural Science Foundation of China (81171421, 61101046).

\section{References}

[1] K. A. MacCannell, H. Bazzazi, L. Chilton, Y. Shibukawa, R. B. Clark, and W. R. Giles, "A mathematical model of electrotonic interactions between ventricular myocytes and fibroblasts," Biophysical Journal, vol. 92, no. 11, pp. 4121-4132, 2007.

[2] F. B. Sachse, A. P. Moreno, G. Seemann, and J. A. Abildskov, "A model of electrical conduction in cardiac tissue including fibroblasts," Annals of Biomedical Engineering, vol. 37, no. 5, pp. 874-889, 2009.

[3] P. Kohl and U. Ravens, "Cardiac mechano-electric feedback: past, present, and prospect," Progress in Biophysics and Molecular Biology, vol. 82, no. 1-3, pp. 3-9, 2003.

[4] N. A. Trayanova and J. J. Rice, "Cardiac electromechanical models: from cell to organ," Frontiers in Physiology, vol. 2, p. 43, 2011.

[5] N. A. Trayanova, "Whole-heart modeling: applications to cardiac electrophysiology and electromechanics," Circulation Research, vol. 108, no. 1, pp. 113-128, 2011.

[6] N. A. Trayanova, J. Constantino, and V. Gurev, "Electromechanical models of the ventricles," American Journal of Physiology. Heart and Circulatory Physiology, vol. 301, no. 2, pp. H279H286, 2011.

[7] R. H. Clayton, O. Bernus, E. M. Cherry et al., "Models of cardiac tissue electrophysiology: progress, challenges and open questions," Progress in Biophysics and Molecular Biology, vol. 104, no. 1-3, pp. 22-48, 2011.

[8] A. Carusi, K. Burrage, and B. Rodriguez, "Bridging experiments, models and simulations: an integrative approach to validation in computational cardiac electrophysiology," American Journal of Physiology. Heart and Circulatory Physiology, vol. 303, no. 2, pp. H144-H155, 2012.

[9] E. M. Cherry and F. H. Fenton, "Effects of boundaries and geometry on the spatial distribution of action potential duration in cardiac tissue," Journal of Theoretical Biology, vol. 285, no. 1, pp. 164-176, 2011.

[10] N. H. Kuijpers, E. Hermeling, P. H. Bovendeerd, T. Delhaas, and F. W. Prinzen, "Modeling cardiac electromechanics and mechanoelectrical coupling in dyssynchronous and failing hearts: insight from adaptive computer models," Journal of Cardiovascular Translational Research, vol. 5, no. 2, pp. 159-169, 2012.

[11] S. A. Niederer and N. P. Smith, "The role of the Frank-Starling law in the transduction of cellular work to whole organ pump function: a computational modeling analysis," PLoS Computational Biology, vol. 5, no. 4, Article ID e1000371, 2009.

[12] S. A. Niederer and N. P. Smith, "An improved numerical method for strong coupling of excitation and contraction models in the heart," Progress in Biophysics and Molecular Biology, vol. 96, no. 1-3, pp. 90-111, 2008.

[13] L. Xia, M. Huo, Q. Wei, F. Liu, and S. Crozier, "Analysis of cardiac ventricular wall motion based on a three-dimensional electromechanical biventricular model," Physics in Medicine and Biology, vol. 50, no. 8, pp. 1901-1917, 2005.

[14] R. C. P. Kerckhoffs, J. H. Omens, A. D. McCulloch, and L. J. Mulligan, "Ventricular dilation and electrical dyssynchrony synergistically increase regional mechanical nonuniformity but not mechanical dyssynchrony: a computational model," Circulation, vol. 3, no. 4, pp. 528-536, 2010.

[15] X.-C. Yang and F. Sachs, "Characterization of stretch-activated ion channels in Xenopus oocytes," Journal of Physiology, vol. 431, pp. 103-122, 1990.

[16] K. Naruse and M. Sokabe, "Involvement of stretch-activated ion channels in $\mathrm{Ca}^{2+}$ mobilization to mechanical stretch in endothelial cells," American Journal of Physiology. Cell Physiology, vol. 264, no. 4, pp. C1037-C1044, 1993.

[17] W. Craelius, V. Chen, and N. El-Sherif, "Stretch activated ion channels in ventricular myocytes," Bioscience Reports, vol. 8, no. 5, pp. 407-414, 1988.

[18] J. O. Bustamante, A. Ruknudin, and F. Sachs, "Stretch-activated channels in heart cells: relevance to cardiac hypertrophy," Journal of Cardiovascular Pharmacology, vol. 17, supplement 2, pp. S110-S113, 1991.

[19] I. Manabe, T. Shindo, and R. Nagai, "Gene expression in fibroblasts and fibrosis involvement in cardiac hypertrophy," Circulation Research, vol. 91, no. 12, pp. 1103-1113, 2002.

[20] V. S. Petrov, G. V. Osipov, and J. Kurths, "Fibroblasts alter spiral wave stability," Chaos, vol. 20, no. 4, Article ID 045103, 2010.

[21] J. M. T. De Bakker and H. M. V. Van Rijen, "Continuous and discontinuous propagation in heart muscle," Journal of Cardiovascular Electrophysiology, vol. 17, no. 5, pp. 567-573, 2006.

[22] M. S. Spach, J. F. Heidlage, P. C. Dolber, and R. C. Barr, "Mechanism of origin of conduction disturbances in aging human atrial bundles: experimental and model study," Heart Rhythm, vol. 4, no. 2, pp. 175-185, 2007.

[23] Y. Xie, A. Garfinkel, P. Camelliti, P. Kohl, J. N. Weiss, and Z. Qu, "Effects of fibroblast-myocyte coupling on cardiac conduction and vulnerability to reentry: a computational study," Heart Rhythm, vol. 6, no. 11, pp. 1641-1649, 2009.

[24] M. A. Rossi, "Connective tissue skeleton in the normal left ventricle and in hypertensive left ventricular hypertrophy and chronic chagasic myocarditis," Medical Science Monitor, vol. 7, no. 4, pp. 820-832, 2001.

[25] M. Miragoli, G. Gaudesius, and S. Rohr, "Electrotonic modulation of cardiac impulse conduction by myofibroblasts," Circulation Research, vol. 98, no. 6, pp. 801-810, 2006.

[26] S. Zlochiver, V. Muñoz, K. L. Vikstrom, S. M. Taffet, O. Berenfeld, and J. Jalife, "Electrotonic myofibroblast-to-myocyte coupling increases propensity to reentrant arrhythmias in twodimensional cardiac monolayers," Biophysical Journal, vol. 95, no. 9, pp. 4469-4480, 2008.

[27] V. Jacquemet and C. S. Henriquez, "Loading effect of fibroblastmyocyte coupling on resting potential, impulse propagation, and repolarization: insights from a microstructure model," 
American Journal of Physiology. Heart and Circulatory Physiology, vol. 294, no. 5, pp. H2040-H2052, 2008.

[28] Y. Xie, A. Garfinkel, J. N. Weiss, and Z. Qu, "Cardiac alternans induced by fibroblast-myocyte coupling: mechanistic insights from computational models," American Journal of Physiology. Heart and Circulatory Physiology, vol. 297, no. 2, pp. H775H784, 2009.

[29] F. B. Sachse, A. P. Moreno, and J. A. Abildskov, "Electrophysiological modeling of fibroblasts and their interaction with myocytes," Annals of Biomedical Engineering, vol. 36, no. 1, pp. 41-56, 2008.

[30] M. Courtemanche, R. J. Ramirez, and S. Nattel, "Ionic mechanisms underlying human atrial action potential properties: insights from a mathematical model," American Journal of Physiology. Heart and Circulatory Physiology, vol. 275, no. 1, part 2, pp. H301-H321, 1998.

[31] N. H. Kuijpers, P. A. J. Hilbers, H. M. M. ten Eikelder, and M. G. J. Arts, Cardiac electrophysiology and mechanoelectric feedback: modeling and simulation [Ph.D. thesis], Technische Universiteit Eindhoven, Eindhoven, The Netherlands, 2008.

[32] J. J. Rice, F. Wang, D. M. Bers, and P. P. De Tombe, "Approximate model of cooperative activation and crossbridge cycling in cardiac muscle using ordinary differential equations," Biophysical Journal, vol. 95, no. 5, pp. 2368-2390, 2008.

[33] M. M. Maleckar, J. L. Greenstein, W. R. Giles, and N. A. Trayanova, "Electrotonic coupling between human atrial myocytes and fibroblasts alters myocyte excitability and repolarization," Biophysical Journal, vol. 97, no. 8, pp. 2179-2190, 2009.

[34] M. P. Nash and A. V. Panfilov, "Electromechanical model of excitable tissue to study reentrant cardiac arrhythmias," Progress in Biophysics and Molecular Biology, vol. 85, no. 2-3, pp. 501-522, 2004.

[35] R. A. Brown, R. Prajapati, D. A. McGrouther, I. V. Yannas, and M. Eastwood, "Tensional homeostasis in dermal fibroblasts: mechanical responses to mechanical loading in threedimensional substrates," Journal of Cellular Physiology, vol. 175, no. 3, pp. 323-332, 1998.

[36] L. Chilton, S. Ohya, D. Freed et al., " $\mathrm{K}^{+}$currents regulate the resting membrane potential, proliferation, and contractile responses in ventricular fibroblasts and myofibroblasts," American Journal of Physiology. Heart and Circulatory Physiology, vol. 288, no. 6, pp. H2931-H2939, 2005.

[37] Y. Shibukawa, E. L. Chilton, K. A. MacCannell, R. B. Clark, and W. R. Giles, " $\mathrm{K}^{+}$currents activated by depolarization in cardiac fibroblasts," Biophysical Journal, vol. 88, no. 6, pp. 3924-3935, 2005.

[38] A. Biernacka and N. G. Frangogiannis, "Aging and cardiac fibrosis," Aging and Disease, vol. 2, no. 2, pp. 158-173, 2011.

[39] C. H. Conrad, W. W. Brooks, J. A. Hayes, S. Sen, K. G. Robinson, and O. H. L. Bing, "Myocardial fibrosis and stiffness with hypertrophy and heart failure in the spontaneously hypertensive rat," Circulation, vol. 91, no. 1, pp. 161-170, 1995.

[40] M. F. Berry, A. J. Engler, Y. J. Woo et al., "Mesenchymal stem cell injection after myocardial infarction improves myocardial compliance," American Journal of Physiology. Heart and Circulatory Physiology, vol. 290, no. 6, pp. H2196-H2203, 2006.

[41] M. Zabel, B. S. Koller, F. Sachs, and M. R. Franz, "Stretchinduced voltage changes in the isolated beating heart: importance of the timing of stretch and implications for stretchactivated ion channels," Cardiovascular Research, vol. 32, no. 1, pp. 120-130, 1996.
[42] M. R. Franz and F. Bode, "Mechano-electrical feedback underlying arrhythmias: the atrial fibrillation case," Progress in Biophysics and Molecular Biology, vol. 82, no. 1-3, pp. 163-174, 2003.

[43] F. Bode, F. Sachs, and M. R. Franz, "Tarantula peptide inhibits atrial fibrillation," Nature, vol. 409, no. 6816, pp. 35-36, 2001.

[44] R. M. Shaw and Y. Rudy, "Electrophysiologic effects of acute myocardial ischemia: a mechanistic investigation of action potential conduction and conduction failure," Circulation Research, vol. 80, no. 1, pp. 124-138, 1997.

[45] V. Jacquemet and C. S. Henriquez, "Modelling cardiac fibroblasts: interactions with myocytes and their impact on impulse propagation," Europace, vol. 9, pp. 29-37, 2007.

[46] S. A. Niederer, P. J. Hunter, and N. P. Smith, "A quantitative analysis of cardiac myocyte relaxation: a simulation study," Biophysical Journal, vol. 90, no. 5, pp. 1697-1722, 2006.

[47] J. Pellman, R. C. Lyon, and F. Sheikh, "Extracellular matrix remodeling in atrial fibrosis: mechanisms and implications in atrial fibrillation," Journal of Molecular and Cellular Cardiology, vol. 48, no. 3, pp. 461-467, 2010.

[48] R. G. Assomull, S. K. Prasad, J. Lyne et al., "Cardiovascular magnetic resonance, fibrosis, and prognosis in dilated cardiomyopathy," Journal of the American College of Cardiology, vol. 48, no. 10, pp. 1977-1985, 2006.

[49] B. T. John, B. K. Tamarappoo, J. L. Titus, W. D. Edwards, W.-K. Shen, and S. S. Chugh, "Global remodeling of the ventricular interstitium in idiopathic myocardial fibrosis and sudden cardiac death," Heart Rhythm, vol. 1, no. 2, pp. 141-149, 2004.

[50] M. L. Antoni, S. A. Mollema, V. Delgado et al., "Prognostic importance of strain and strain rate after acute myocardial infarction," European Heart Journal, vol. 31, no. 13, pp. 16401647, 2010.

[51] P. Kohl, P. Camelliti, F. L. Burton, and G. L. Smith, "Electrical coupling of fibroblasts and myocytes: relevance for cardiac propagation," Journal of Electrocardiology, vol. 38, no. 4, supplement, pp. 45-50, 2005.

[52] M. B. Rook, A. C. G. Van Ginneken, B. De Jonge, A. El Aoumari, D. Gros, and H. J. Jongsma, "Differences in gap junction channels between cardiac myocytes, fibroblasts, and heterologous pairs," American Journal of Physiology. Cell Physiology, vol. 263, no. 5, pp. C959-C977, 1992.

[53] P. Kohl, A. G. Kamkin, I. S. Kiseleva, and D. Noble, "Mechanosensitive fibroblasts in the sino-atrial node region of rat heart: interaction with cardiomyocytes and possible role," Experimental Physiology, vol. 79, no. 6, pp. 943-956, 1994. 


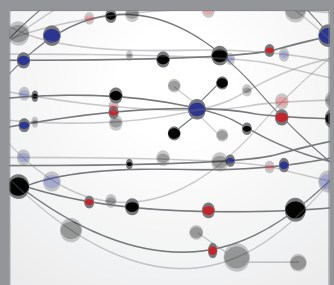

The Scientific World Journal
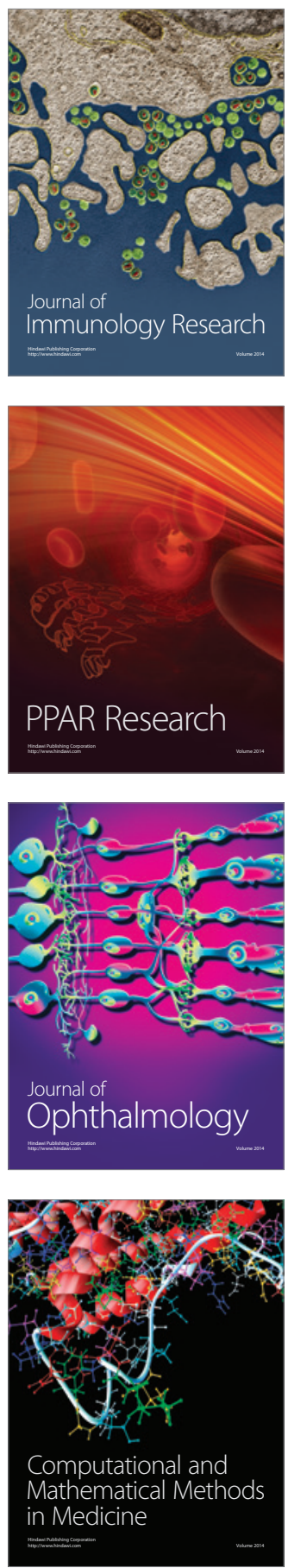

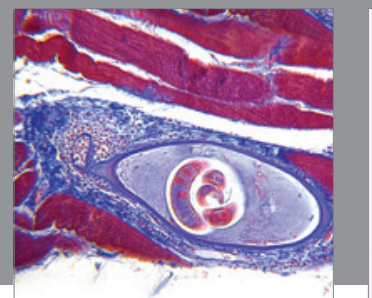

Gastroenterology

Research and Practice
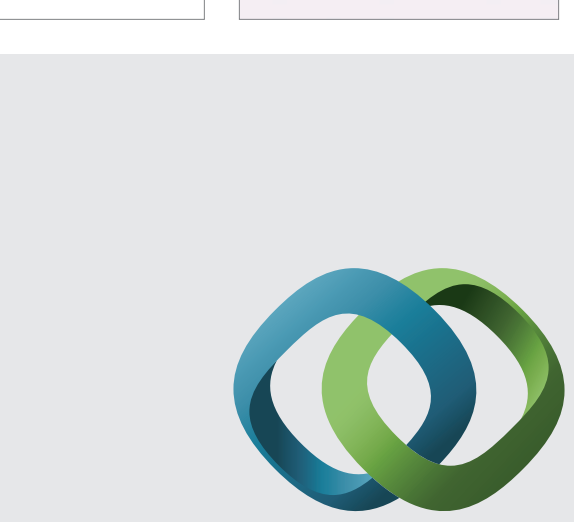

\section{Hindawi}

Submit your manuscripts at

http://www.hindawi.com
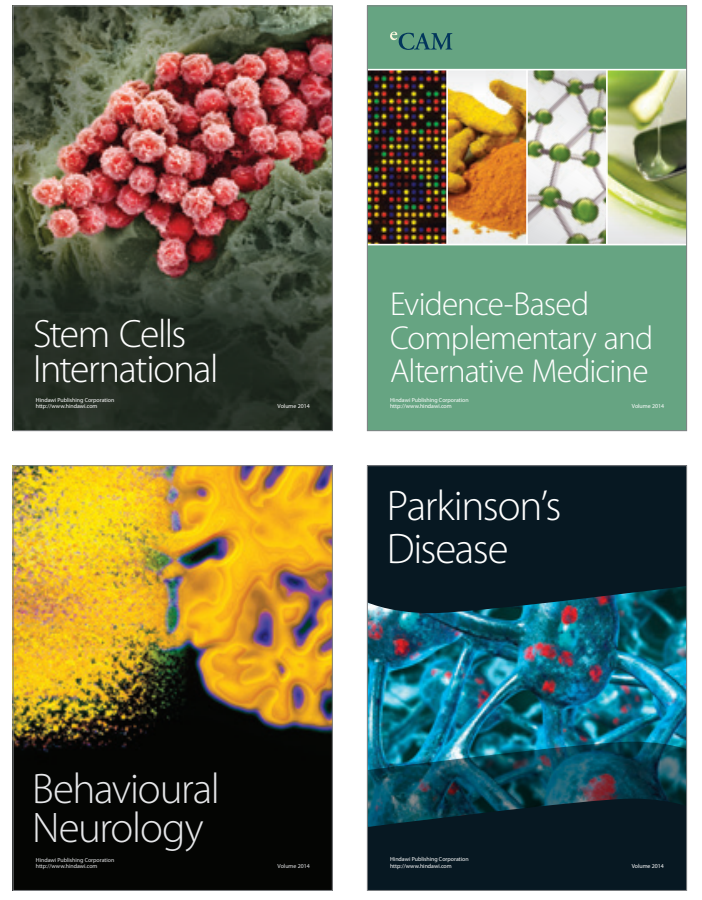
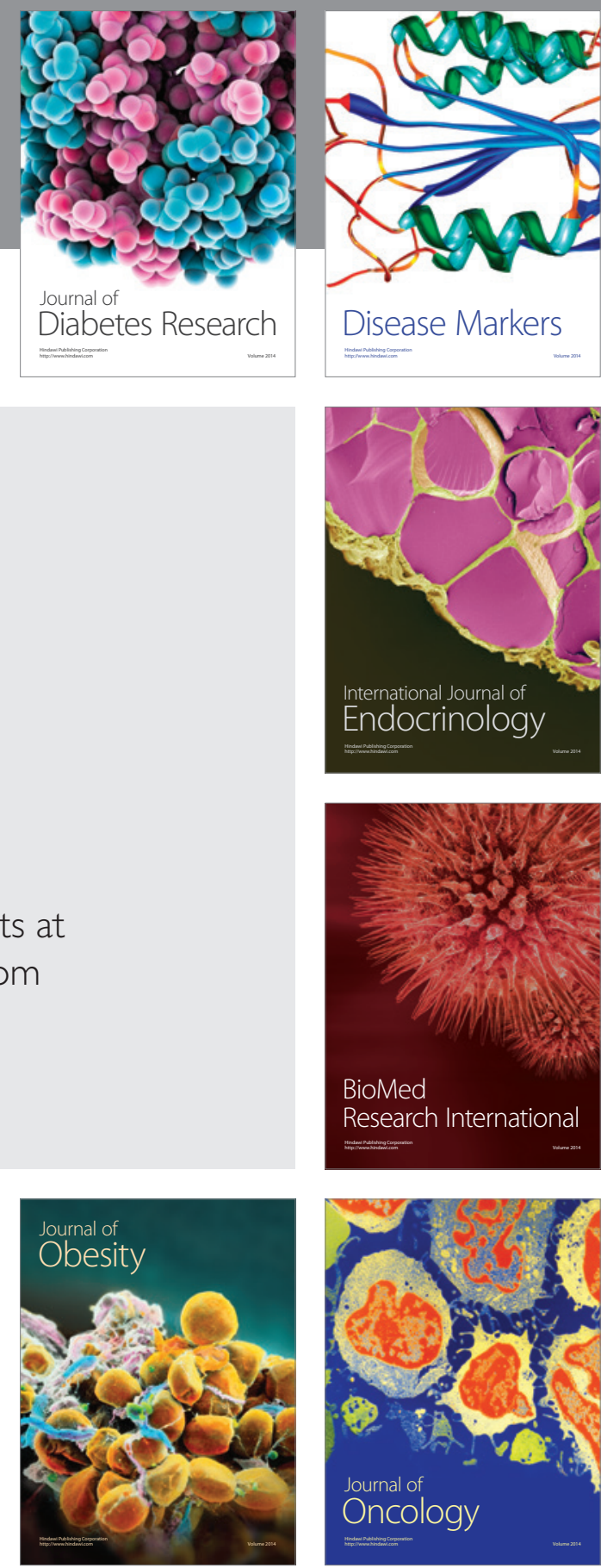

Disease Markers
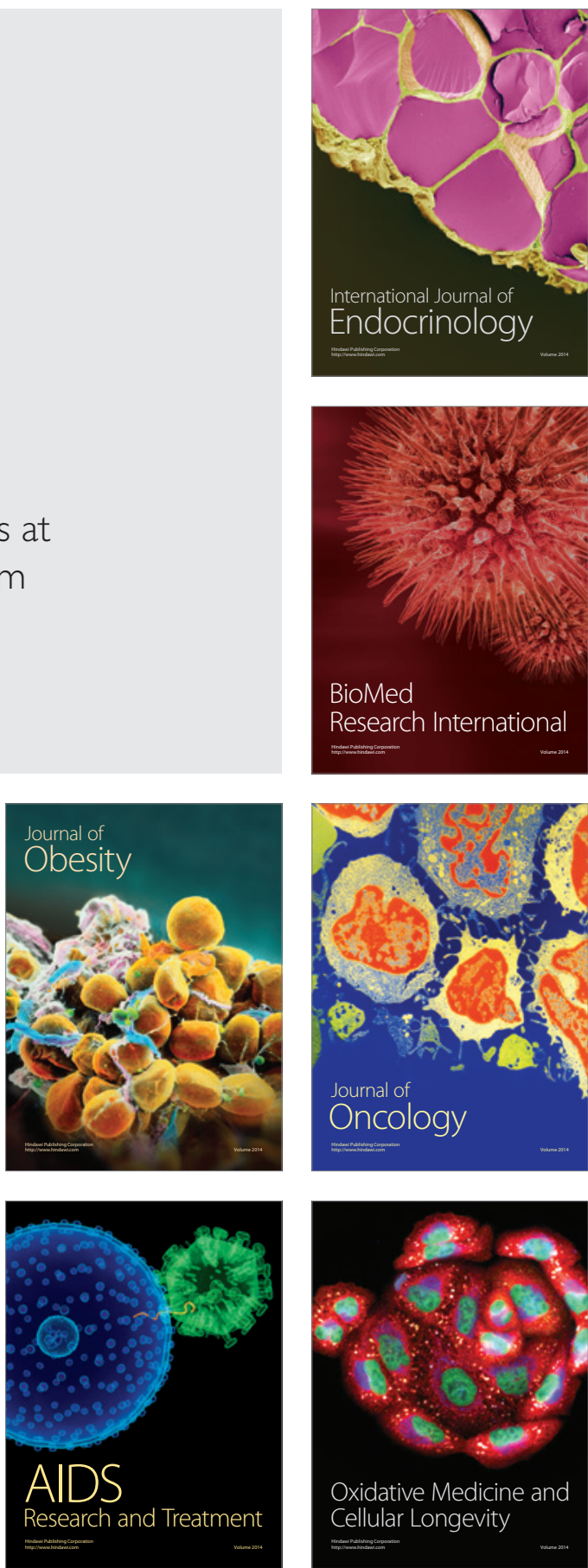